MZ-TH/94-07

PLY-MS-94-06

\title{
Perturbative and Non-Perturbative Quarks and Gluons
}

\author{
Martin LaVElle ${ }^{* 1}$ AND David MCMullan ${ }^{\dagger 2}$ \\ *Institut für Physik \\ Johannes Gutenberg-Universität \\ Staudingerweg 7, Postfach 3980 \\ D-55099 Mainz, F.R. Germany \\ †School of Mathematics and Statistics \\ University of Plymouth \\ Drake Circus, Plymouth, Devon PL4 8AA \\ U.K.
}

\begin{abstract}
BRST invariance supplies a sufficient condition for the observability of fields. We show that there is a global obstruction to the observability of quarks and gluons and argue that they will not become observables at finite temperature. We give expressions for quarks and gluons that are, however, perturbatively BRST invariant, and hence locally observable, up to order $g^{2}$ and $g$ respectively.
\end{abstract}

\footnotetext{
1 e-mail: lavelle@vipmza.physik.uni-mainz.de

2 e-mail: d.mcmullan@plymouth.ac.uk
} 
Quantum Chromodynamics (QCD) is the theory of strong interactions ${ }^{[1]}$. Its success is based on perturbation theory. The content of the theory is a non-abelian, $S U(3)$, interaction of quarks and gluons. Evidence for these particles comes from deep inelastic scattering. The outstanding problem in QCD is that these particles have not been observed experimentally. This has led to the confinement hypothesis that only colour singlet objects are observed in nature. In this letter we will prove that it is impossible to construct an observable quark or gluon outside of perturbation theory. Inside perturbation theory, however, this may be done. We present expressions for such perturbatively observable quarks and gluons to low orders in the coupling.

The QCD Lagrangian is

$$
\mathcal{L}=-\frac{1}{4} F^{2}+\bar{\psi}(i \not D-m) \psi
$$

where $F$ is the field strength constructed out of the non-abelian (Lie algebra valued) potentials $A, D$ is the associated covariant derivative and $\psi$ is a fermionic field. This Lagrangian exhibits the following gauge invariance

$$
\begin{aligned}
& A(x) \rightarrow A^{U}(x)=U^{-1}(x) A(x) U(x)+\frac{1}{g} U^{-1}(x) d U(x), \\
& \psi(x) \rightarrow \psi^{U}(x)=U^{-1}(x) \psi(x)
\end{aligned}
$$

where $g$ is the coupling and, for each $x, U(x)$ is an element of $\mathrm{SU}(3)$ (or more generally any compact Lie group). Due to the existence of constraints, which is a direct consequence of this gauge invariance, observables must be gauge (or, more exactly, BRST) invariant. From Eq. 2 we see that, in particular, the fermionic fields, $\psi$ and $\bar{\psi}$, are not observables and thus cannot be identified with observable quarks. A similar problem for the electron arises in QED and has been solved by Dirac ${ }^{[2]}$ as we now explain (see also Ref. 3).

The physical electron field is given by

$$
\psi_{\text {phys }}(x)=\exp \left(i g \frac{\partial_{i} A_{i}}{\nabla^{2}}(x)\right) \psi(x) \text {. }
$$

This is a spatially non-local field, with $\frac{1}{\nabla^{2}}$ being the Green's function for the Laplacian $\nabla^{2}=\partial_{i} \partial_{i}$. This type of non-locality in the electron field is perfectly acceptable and reflects the need for an infinite number of soft photons to deal with the infra-red sector of the theory ${ }^{[4]}$. Indeed, in contrast to the usual asymptotic identification of the electron with 
$\psi$, this BRST invariant expression has an electromagnetic charge and creates a Coulomb electric field ${ }^{[2]}$; justifying its identification with the electron.

In contrast to this, the gauge and BRST invariant expression

$$
\psi_{\text {cov }}(x)=\exp \left(-i g \frac{\partial_{\mu} A^{\mu}(x)}{\square^{2}}\right) \psi(x),
$$

cannot be identified with a physical field due to the spacetime, and in particular temporal, non-locality implicit in the $\frac{1}{\square^{2}}$ Green's function. Being non-local in time obstructs any decomposition into positive and negative frequency components and hence a particle picture. Indeed the whole concept of in and out states, as well as time ordering, loses any meaning for this field. This example, though, does show the need for the caveat that BRST invariance is only a sufficient condition for the observability of a given field.

Another possible candidate for an electron would be

$$
\psi_{3}(x)=\exp \left(-i g \frac{A_{3}(x)}{\partial_{3}}\right) \psi(x)
$$

Although this is naively gauge invariant, it depends upon an arbitrary direction which is unattractive in such a physical field. Indeed, a more detailed investigation ${ }^{[5]}$ shows that this field configuration is unstable, and decays into the static expression (3) proposed by Dirac.

An at first sight distinctly different ansatz is as follows. Consider a fermion attached to a Wilson line

$$
\psi_{\Gamma}(x)=\exp \left(i g \int_{-\infty}^{x} A_{\mu}(z) d z^{\mu}\right) \psi(x),
$$

where $\Gamma$ is any contour from the point $x$ to $-\infty$. Although this is, by construction, gauge invariant, it is dependent on the arbitrary line $\Gamma$. A physical electron cannot have this property. We now require that the contour does not introduce any temporal non-locality. Then, upon decomposing the spatial components into the physical, transverse components, $A_{i}^{T}$, and the unphysical, longitudinal component, $A_{i}^{L}=\frac{\partial_{i} \partial_{j} A_{j}}{\nabla^{2}}$, we see that $\psi_{\Gamma}$ may be written as

$$
\psi_{\Gamma}(x)=N_{\Gamma}(x) \psi_{\text {phys }}(x),
$$

where

$$
N_{\Gamma}(x)=\exp \left(i g \int_{-\infty}^{x} A_{i}^{T}(z) d z^{i}\right)
$$


This gauge invariant normalisation factor contains all the contour dependence and must be removed for the fermion to have any physical meaning. We thus recover Dirac's physical electron. Indeed it should be noted that if, as was suggested in Ref. 6, we replace $A_{\mu}(z) d z^{\mu}$ by $A_{i}^{L}(z) d z^{i}$, we obtain just Dirac's electron (since $N_{\Gamma}$ is then unity as may be seen from Eq. 8). This concludes our discussion of QED for the moment.

A sufficient condition for the non-observability of quarks would be to show that no contour and gauge independent generalisation of Dirac's physical electron can be constructed for the quarks. We will now demonstrate that this is the case.

Working in a Hamiltonian description ${ }^{[7]}$ of $\mathrm{QCD}$, where the momentum conjugate to the potential is denoted by $\Pi(x)$, i.e., such that the fundamental Poisson brackets are $\left\{A_{i}^{a}(x), \Pi_{b}^{j}(y)\right\}=\delta_{b}^{a} \delta_{i}^{j} \delta(x-y)$, we see that if such a field exists it may be written as

$$
\psi_{\text {phys }}(x)=h^{-1}(x) \psi(x)
$$

where $h(x)$ is a field dependent element of $S U(3)$. The sought for gauge invariance of this expression then implies that, under a gauge transformation, $h$ must transform as

$$
h(x) \rightarrow h^{U}(x)=U^{-1}(x) h(x) .
$$

Thus writing

$$
h(x)=\exp \left(-v^{a}(x) T^{a}\right),
$$

where $T^{a}$ denotes the (here chosen to be anti-Hermitian) Gell-Mann matrices, and expanding the exponential we find that the infinitesimal version of (10) is

$$
\left\{v^{b}(x), G^{a}(y)\right\} \approx \delta^{a b} \delta(x-y)
$$

where $G^{a}$ is the infinitesimal generator of gauge transformations

$$
G^{a}(x)=\left(D_{i} \Pi^{i}\right)^{a}(x)+g J_{0}^{a}(x),
$$

and $J_{0}^{a}$ is the current density. Note that we are using a weak equivalence in (12), i.e., it holds only after we have set the constraints and $v^{a}(x)$ to zero. Thus, if such a construction were possible, we could identify $v^{a}(x)$ as possible gauge fixing functions. (In QED we have seen that it is essentially the Coulomb gauge.) We now assume that our fields are 
chosen so that, as far as the gauge group is concerned, we can identify the space time with $\mathbb{R} \times S^{3}$, where $S^{3}$ is the spatial three sphere. As is well known ${ }^{[8]}$ there is no such global gauge fixing in QCD (the Gribov ambiguity ${ }^{3}$ ). Hence we deduce that there is no gauge invariant description of a single quark ${ }^{[11]}$. Of course there are observables in $\mathrm{QCD}$, these correspond to gauge invariant combinations of the fundamental fields; an example is $\bar{\psi} \psi$. We would like to stress that our arguments do not depend upon working in a particular gauge or indeed upon using a gauge fixing at all: we merely note that if physical quarks could be defined then they could be used to construct a globally well defined gauge fixing. Since this last does not exist, neither do observable quarks.

Quark fields have not been directly observed in experiments and this has led to the confinement hypothesis, i.e., that they never will be. We now argue that the non-observability of quark fields provides an explanation for confinement. Indeed if we assume to the contrary that quarks are not confined then they must be directly observed in experiments. In terms of the structure of $\mathrm{QCD}$, what this would mean is that we can construct a one quark state (in some asymptotic region). However, we have shown that it is not possible to construct such a state - thus we have a contradiction unless quarks are confined.

We stress that the above is a sufficient condition for confinement, and is not a necessary one. Indeed, abelian theories (for example, compact $U(1)$ in three dimensions ${ }^{[12]}$ ) may also confine due to dynamical effects.

The above simple argument for confinement cannot be considered watertight - it skims over many difficult epistemological questions that plague field theory and indeed quantum mechanics. On a more immediate level, it fails to convey any scale for confinement. Before starting to address this important topic, though, let us briefly discuss the analogous problem of the observability (or not) of the gluonic field.

Just as we saw for the fermionic field, the gauge field $A$ cannot be identified with the physical gluons due to the gauge transformation (2). However, if we could construct the $\mathrm{SU}(3)$-valued field $h$, transforming as in (10), then we claim that the physical gluonic field can be identified with the $h$-transformed potential

$$
A_{\text {phys }}:=A^{h}=h^{-1} A h+\frac{1}{g} h^{-1} d h .
$$

3 It is sometimes argued that algebraic gauges do not suffer from the ambiguity. They are, however, afflicted with many other diseases, see, e.g., Ref. 9. In particular these gauge choices are incompatible with the physical photons ${ }^{[10]}$. 
Indeed, under a gauge transformation we have

$$
\begin{aligned}
A_{\mathrm{phys}} & \rightarrow\left(A^{U}\right)^{U^{-1} h} \\
& =\left(U^{-1} h\right)^{-1} A^{U} U^{-1} h+\frac{1}{g}\left(U^{-1} h\right)^{-1} d\left(U^{-1} h\right) \\
& =h^{-1} U A^{U} U^{-1} h+\frac{1}{g} h^{-1}\left(U d U^{-1}\right) h+\frac{1}{g} h^{-1} d h \\
& =h^{-1}\left(A+\frac{1}{g} d U U^{-1}\right) h-\frac{1}{g} h^{-1}\left(d U U^{-1}\right) h+\frac{1}{g} h^{-1} d h \\
& =A^{h} .
\end{aligned}
$$

So we see that knowledge of $h$ suffices to specify the physical matter and gauge fields in a gauge theory. There is an appealing economy in this description of the physical fields that is only marred by the global lack of existence of such a field in the non-abelian theory. However, we can immediately deduce that in QCD the gluonic field is also not observable. For the abelian theory we have seen that we can take

$$
h=\exp \left(-g \frac{\partial_{i} A_{i}}{\nabla^{2}}\right)
$$

which yields the physical electron (3) and from (14) gives

$$
A_{\mathrm{phys}}^{i}=\left(\delta^{i j}-\frac{\partial^{i} \partial^{j}}{\nabla^{2}}\right) A_{j},
$$

the two transverse photons. (Indeed, had we used the axial type of prescription found in (5), then the corresponding photon field would not be transverse. The additional longitudinal components can be seen to be responsible for the radiative decay of (5) discussed in Ref. 5.)

Although, as seen above, no global solution to (10) exists in a non-abelian theory, we may attempt to find a local solution. It is important to stress here that local means local in the Yang-Mills configuration space, which is not directly related to the spatial locality we want in order to extract a confining scale. Indeed there are no natural scales in classical QCD, thus we can only hope to start quantifying our account of confinement if we can demonstrate that a sensible perturbative expansion of the physical fields (3) and (14) exists in some region of the configuration space. We are looking for a local solution in terms of a power series in the coupling $g$. In this way we may define perturbatively physical quarks and gluons. 
The Lagrangian fermion is invariant up to order $g^{0}$ reflecting the fact that the fermion is a physical particle when the gauge interaction is switched off. (This is of course not true of the gauge fields even in the free photon theory.) When we switch on the gauge interaction we have to dress the fermion. A perturbative quark field which is invariant up to order $g^{1}$ is seen by inspection to be

$$
\psi_{\mathrm{phys}}^{g^{1}}(x)=\left(1+g \frac{\partial_{i} A_{i}^{a}}{\nabla^{2}}(x) T^{a}\right) \psi(x)
$$

which we may suggestively rewrite as

$$
\psi_{\text {phys }}^{g^{1}}(x)=\exp \left(g \frac{\partial_{i} A_{i}^{a}}{\nabla^{2}}(x) T^{a}\right) \psi(x)+\mathcal{O}\left(g^{2}\right)
$$

i.e., to this level in the coupling we reproduce the Dirac electron. (We must merely replace $A_{i}$ by $A_{i}^{a} T^{a}$.) Equivalently we have

$$
h^{g^{1}}(x)=\exp \left(-g \frac{\partial_{i} A_{i}^{a}}{\nabla^{2}}(x) T^{a}\right)+\mathcal{O}\left(g^{2}\right) .
$$

From this last equation together with (14) we see that

$$
\left(A_{\mathrm{phys}}^{g^{0}}\right)^{i}(x)=\left(\delta^{i j}-\frac{\partial^{i} \partial^{j}}{\nabla^{2}}\right) A_{j}(x)
$$

i.e., to lowest order in the coupling we again obtain the QED result. It should be noted that due to the factors of $\frac{1}{g}$ in (14) and (2) knowledge of $h$ to any particular power in the coupling only suffices to specify the perturbatively physical gluons up to one power less in $g$.

With the above fields we may perform some lowest order perturbation theory. The one-loop perturbative quark propagator is just the one loop physical electron propagator, which was studied in Ref. 4, up to an unimportant colour factor. This is gauge invariant and is the usual one-loop perturbative fermion propagator in Coulomb gauge. Indeed the perturbatively physical fields generated by $h^{g^{1}}$ reduce to the standard ones in Coulomb gauge as may be seen from (19) and (21). 
We now want to go on to determine $h$ to order $g^{2}$. After some algebra one finds that

$$
\begin{aligned}
h^{g^{2}}(x)= & -g \frac{\partial_{i} A_{i}^{a}}{\nabla^{2}}(x) T^{a}+\frac{g^{2}}{2}\left(\frac{\partial_{i} A_{i}^{a}}{\nabla^{2}}(x) T^{a}\right)^{2} \\
& +\frac{g^{2} f^{a b c}}{2} \frac{1}{\nabla^{2}}\left(A_{j}^{b} \frac{1}{\nabla^{2}} \partial_{j} \partial_{i} A_{i}^{c}\right)(x) T^{a}+\frac{g^{2} f^{a b c}}{2} \frac{1}{\nabla^{2}} \partial_{j}\left(A_{j}^{b} \frac{1}{\nabla^{2}} \partial_{i} A_{i}^{c}\right)(x) T^{a}, \\
= & \exp \left\{\left(-g \frac{\partial_{i} A_{i}^{a}}{\nabla^{2}}(x)+\frac{g^{2} f^{a b c}}{2} \frac{1}{\nabla^{2}}\left(A_{j}^{b} \frac{1}{\nabla^{2}} \partial_{j} \partial_{i} A_{i}^{c}\right)(x)\right.\right. \\
& \left.\left.+\frac{g^{2} f^{a b c}}{2} \frac{1}{\nabla^{2}} \partial_{j}\left(A_{j}^{b} \frac{1}{\nabla^{2}} \partial_{i} A_{i}^{c}\right)(x)\right) T^{a}\right\}+\mathcal{O}\left(g^{3}\right),
\end{aligned}
$$

fulfills this requirement up to terms of order $g^{3}$. With this $h^{g^{2}}$ we find the following perturbatively physical fields:

$$
\begin{aligned}
\psi_{\text {phys }}^{g^{2}}(x)=(1 & +g \frac{\partial_{i} A_{i}^{a}}{\nabla^{2}}(x) T^{a}+\frac{g^{2}}{2}\left(\frac{\partial_{i} A_{i}^{a}}{\nabla^{2}}(x) T^{a}\right)^{2} \\
& -\frac{g^{2} f^{a b c}}{2} \frac{1}{\nabla^{2}}\left(A_{j}^{b} \frac{1}{\nabla^{2}} \partial_{j} \partial_{i} A_{i}^{c}\right)(x) T^{a} \\
& \left.-\frac{g^{2} f^{a b c}}{2} \frac{1}{\nabla^{2}} \partial_{j}\left(A_{j}^{b} \frac{1}{\nabla^{2}} \partial_{i} A_{i}^{c}\right)(x) T^{a}\right) \psi(x),
\end{aligned}
$$

and

$$
\begin{aligned}
\left(A_{\mathrm{phys}}^{g^{1}}\right)_{i}(x)= & A_{i}(x)-\frac{\partial_{i} \partial_{j} A_{j}}{\nabla^{2}}(x) \\
& +g f^{a b c} T^{a}\left[\left(\frac{\partial_{j} A_{j}^{b}}{\nabla^{2}}\right)(x) A_{i}^{c}(x)+\frac{1}{2}\left(\frac{\partial_{j} A_{j}^{b}}{\nabla^{2}}\right)(x)\left(\frac{\partial_{i} \partial_{k} A_{k}^{c}}{\nabla^{2}}\right)(x)\right. \\
& \left.+\frac{1}{2} \frac{1}{\nabla^{2}} \partial_{i}\left(A_{j}^{b} \frac{1}{\nabla^{2}}\left(\partial_{j} \partial_{k} A_{k}^{c}\right)\right)(x)+\frac{1}{2} \frac{1}{\nabla^{2}} \partial_{i} \partial_{j}\left(A_{j}^{b} \frac{1}{\nabla^{2}}\left(\partial_{k} A_{k}^{c}\right)\right)(x)\right] .
\end{aligned}
$$

Note that they both reduce to the usual fields in Coulomb gauge and that the physical gluonic field is transverse to this order, i.e., $\partial_{i}\left(A_{\text {phys }}^{g^{1}}\right)^{i}=0$. It may be straightforwardly checked that these fields are indeed invariant under both BRST and gauge transformations up to terms of order $g^{3}$ and $g^{2}$ respectively.

There have been previous attempts ${ }^{[13-16]}$ to find the true degrees of freedom in pure $S U(2)$ Yang-Mills theory. These solutions have displayed unpleasant singularities and have not proven to be very tractable. The suspicion has been voiced ${ }^{[16]}$ that there exists 
a link between these singularities and the Gribov ambiguity. From the above results it is clear that this is indeed the case: due to the Gribov ambiguity it is impossible to find physical quarks or gluons and the singularities reflect this. It is however, possible to find perturbative solutions and this we have done here. The field theorists standard tools may now be applied to these solutions.

The results presented here should be used as the basis of a perturbative investigation of the physical Green's functions of QCD which, as we have discussed above, is essential if the scale of confinement is to emerge from this approach (now being identified with the breakdown of gauge invariance in the locally physical Green's functions). On top of this, it would be of interest to see what light they cast upon the infra-red behaviour of the theory; recall that in QED the infra-red singularities cancel in the physical Green's functions ${ }^{[4]}$. The higher order extension of the solutions found here should also be investigated. We remark in this context that problems with the Coulomb gauge at three loops have been seen by Taylor ${ }^{[17]}$.

The topological account of the inevitability of the Gribov ambiguity in non-abelian gauge theories fails to give a feel for the size of the obstruction. One knows that it is not just the large gauge transformations that are causing problems. In fact, within the identity component of the group of all gauge transformations there are non-contractable loops (for $\mathrm{SU}(2)$ ), spheres and higher dimensional objects all conspiring to stop the local observables from becoming global. However, without any metric information we cannot decide whether such topological complications cause trouble in a large region of the configuration space or just at a few isolated points (after all, an $n$-sphere minus one point is topologically trivial). Clearly the need to assess the extent of the obstruction is related to the spacetime confining scale, and is also important for any consideration of the (local) stability of the solutions. We have seen that the local expressions for the quark and gluon, at least to low order in $g$, are closely connected to Yang-Mills configurations in the Coulomb gauge. Now it is known ${ }^{[18]}$ that the Yang-Mills fields which satisfy this gauge condition can be identified with the critical points of a naturally defined Morse function constructed out of an $L^{2}$-norm on the configuration space. We would thus expect this norm to be of some use in the analysis of the extent to which the fields remain physical as they are extended to the Gribov horizon.

In order to describe QCD at finite temperature and density our assumptions on the topology of space time must be replaced by $S^{1} \times S^{3}$. This additional complication of 
the topology will not alter our arguments, thus we predict that quarks and gluons will not become observables at high temperature. The scales will change of course and it is possible that a large plasma may be generated in which parton-type objects may appear to move around, analogously to the situation in deep inelastic scattering, however, individual quarks or gluons will still not be observables ${ }^{[19]}$.

Another kinematical account of confinement has been proposed by Kugo and Ojima ${ }^{[20]}$. The connection between their work and ours is unclear to us, in particular they make no reference to the role of the Gribov ambiguity. Ideas which appear closer to those advanced here may be found in Ref. 21. Also worth noting here is the idea of Polonyi to link quark confinement to topological obstacles to constructing a gauge invariant quark propagator $^{[22]}$. That this cannot be done is also, if on different grounds, an immediate consequence of our above arguments. Finally we recall that there have been attempts ${ }^{[23,24]}$ to derive confinement dynamically from the Gribov ambiguity, although these papers did not concern themselves with the true degrees of freedom of QCD.

To summarise: we have shown that it is impossible to construct physical, BRST invariant quarks and gluons as a consequence of the Gribov ambiguity. We interpret this as meaning that quarks and gluons are not observable, which is one way of stating the confinement hypothesis. We have, however, been able to construct solutions inside perturbation theory which are BRST invariant to low orders in the coupling. The one-loop perturbatively physical quark propagator was seen to be a close copy of the physical electron propagator and is hence gauge invariant and infra-red finite. A detailed perturbative study of the higher order solutions found here will be presented elsewhere.

Acknowledgements MJL thanks the Graduierten Kolleg of Mainz University for support. 


\section{References}

[1] T. Muta, "Foundations of Quantum Chromodynamics", (World Scientific, Singapore, 1987).

[2] P.A.M. Dirac, "Principles of Quantum Mechanics", (OUP, Oxford, 1958), page 302.

[3] M. Lavelle and D. McMullan, Phys. Rev. Lett. 71 (1993) 3758.

[4] M. Lavelle and D. McMullan, Phys. Lett. 312B (1993) 211.

[5] S.V. Shabanov, "The Proper Field of Charges and Gauge Invariant Variables in Electrodynamics", Dubna preprint, JINR-E2-92-136.

[6] T. Kashiwa and Y. Takahashi, "Gauge Invariance in Quantum Electrodynamics", Kyushu and Alberta preprint KYUSHU-HET-14 (1994).

[7] R. Jackiw, in "Current Algebra and Anomalies", by S.B. Treiman et al. (World Scientific, Singapore, 1987).

[8] V.N. Gribov, Nucl. Phys. B139 (1978) 1;

I. Singer, Commun. Math. Phys. 60 (1978) 7.

[9] H. Cheng and E.C. Tsai, Phys. Rev. D34 (1986) 3858; M. Lavelle and D. McMullan, Z. Phys. C59 (1993) 351.

[10] M. Lavelle and D. McMullan, Phys. Lett. 316B (1993) 172.

[11] See also, M. Lavelle and D. McMullan, "On Quark Confinement", Dublin and Mainz preprint DIAS-STP-93-04 and MZ-TH/93-03, unpublished.

[12] A. Polyakov, "Gauge Fields and Strings", (Harwood, Chur, 1987).

[13] J. Goldstone and R. Jackiw, Phys. Lett. 74B (1978) 81.

[14] V. Baluni and B. Grossman, Phys. Lett. 78B (1978) 226.

[15] A.G. Izergin et al, Theor. Math. Phys. (1979) 3.

[16] A. Das, M. Kaku and P.K. Townsend, Nucl. Phys. B149 (1979) 109.

[17] J.C. Taylor, in proceedings of the workshop "Physical and Nonstandard Gauges", Ed.'s P. Gaigg et al (Springer-Verlag, Berlin, 1990).

[18] G. Dell'Antonio and D. Zwanziger, Commun. Math. Phys. 138 (1991) 291; P. van Baal, Nucl. Phys. B369 (1992) 259.

[19] We thank David Seibert for correspondence on this point.

[20] See, for example, the discussion in N. Nakanishi and I. Ojima, "Covariant Operator Formalism of Gauge Theories and Quantum Gravity", (World Scientific, Singapore, 1990).

[21] S.V. Shabanov, Mod. Phys. Lett. A6 (1991) 909; Phys. Lett. 255B (1991) 398;

L.V.Prokhorov and S.V. Shabanov, Int. J. Mod. Phys. A7 (1992) 7815.

[22] J. Polonyi, Phys. Lett. 213B (1988) 340.

[23] C.M. Bender, T. Eguchi and H. Pagels, Phys. Rev. D17 (1978) 1086.

[24] R.D. Peccei, Phys. Rev. D17 (1978) 1097. 